\title{
Synchronization in ecological systems by weak dispersal coupling with time delay
}

\author{
Emily Wall • Frederic Guichard • Antony R. \\ Humphries
}

Received: date / Accepted: date

\begin{abstract}
One of the most salient spatio-temporal patterns in population ecology is the synchronization of fluctuating local populations across vast spatial extent. Synchronization of abundance has been widely observed across a range of spatial scales in relation to rate of dispersal among discrete populations. However, the dependence of synchrony on patterns of among-patch movement across heterogeneous landscapes has been largely ignored. Here we consider the duration of movement between two predator-prey communities connected by weak dispersal, and its effect on population synchrony. More specifically, we introduce time delayed dispersal to incorporate the finite transmission time between discrete populations across a continuous landscape. Reducing the system to a phase model using weakly connected network theory, it is found that the time delay is an important factor determining the nature and stability of phase-locked states. Our analysis predicts enhanced convergence to stable synchronous fluctuations in general, and a decreased ability of systems to produce in-phase synchronization dynamics in the presence of delayed dispersal. These results introduce delayed dispersal as a tool for understanding the importance of dispersal time across a landscape matrix in affecting metacommunity dynamics. They further highlight the importance of landscape and dispersal patterns for predicting the onset of synchrony between weakly-coupled populations.
\end{abstract}

Keywords Synchronization · Phase model · Dispersal · Time delay

\section{Introduction}

Predicting the onset and maintenance of synchronous fluctuations of abundance among populations has led to important progress in the understanding of population persistence, species coexistence, and response to environmental change. However, most theoretical studies of synchrony have assumed instantaneous and passive movement of individuals among discrete habitats. In natural systems, discrete populations are typically embedded within a landscape matrix that constrains patterns and duration of movement between populations. It is thus important to predict the impact of such a landscape matrix on the emergence and stability of synchronous fluctuations of abundance between distant populations. Here we incorporate delayed dispersal as a means to study landscape and dispersal patterns between discrete predator-prey communities.

Synchronization is generally understood as a phenomenon where through interactions with one another, the timings of fluctuations in components of a dynamical system lock to a single pattern of variation in time. This concept arises in a variety of systems in physics, chemistry, social sciences, and biology: from crickets chirping in synchrony, to fireflies able to all flash in unison (Blasius and Tönjes 2007). Spatial

E. Wall · F. Guichard

Department of Biology, McGill University, Montreal, Canada

E-mail: emily.wall@mail.mcgill.ca

A.R. Humphries

Department of Mathematics and Statistics, McGill University, Montreal, Canada 
synchronization in the context of population ecology refers to coincident changes in population characteristics such as abundance, reproduction, mortality, or mean size or age distribution, in geographically separated populations (Liebhold et al. 2004). Ecological examples of synchronization include the cycling of hare-lynx populations, where fluctuations of groups across Canada were found to be locked on the same tight ten-year cycle (Blasius and Tönjes 2007). Synchronized population dynamics have been observed in natural systems across a variety of taxa and spatial scales, from fungal plant pathogens across $0.5-3 \mathrm{~m}$, insect herbivores across 1-1000km, to birds across 5-2000km (Liebhold et al. 2004).

For our purpose we study the synchrony of coupled oscillators, where individual populations undergo intrinsic oscillations in abundance with some natural frequency, and adjust their oscillations due to coupling (dispersal) between oscillators. This formalism has been used to predict the onset of synchrony in simple ecological systems (Goldwyn and Hastings 2008, Goldwyn and Hastings 2011, Bresloff and Lai 2012). Networks of time-delayed and weakly connected oscillators have also been well-studied in physics, engineering, and neurophysiology (Schuster and Wagner 1989, Dhamala et al. 2004, Campbell and Kobelevskiy 2012). However, no study has elucidated the role of delayed dispersal in coupled ecological systems.

Causes of spatial synchrony have been studied extensively across systems and scales. Coupled oscillator theory predicts that oscillators can be synchronized through direct coupling, or by external forcing. Equivalently in ecology, there are two main mechanisms that produce synchrony: $(a)$ density-dependent direct interactions, namely dispersal between populations, and (b) what is known as the Moran effect (Moran 1953), which is the entrainment of systems with similar density-dependent dynamical structures by correlated density-independent external factors, such as climate or weather (Liebhold et al. 2004). Because most natural systems are connected through both dispersal and external stochastic fluctuations, and because both mechanisms produce similar patterns of synchrony it is often difficult to determine which factor or combination of factors underlies observed patterns of spatial synchrony.

Theory predicts that populations oscillating due to the same or slightly different density-dependent processes (linear or nonlinear) can be synchronized by dispersal of just a very small number of individuals per generation (Liebhold et al. 2004). However, if density-dependent processes are so different that populations oscillate with very different frequencies, then synchronization may not be possible through dispersal (Ranta et al. 1998). Besides dynamical stability of synchronization fluctuations, the rate of synchronization is another constraint on the ability of dispersal to explain synchrony in natural populations: dispersal-induced synchrony must converge rapidly to be observed (Goldwyn and Hastings 2008). Otherwise, populations will be kept in transient non-synchronous states by environmental perturbations. While strong coupling (i.e., a large number of individuals disperse per generation) can always increase local convergence rates to synchrony in general, in a two predator-prey patch system connected only weakly by dispersal, the required property for fast convergence to synchrony is the separation on time scales between predator and prey dynamics (Goldwyn and Hastings 2008). This property is characteristic of relaxation oscillators where a large part of the cycle is spent at low population densities. In contrast, sinusoidal oscillators take a very long time to synchronize by weak dispersal. Our study examines some mechanisms underlying this result and uses its robustness to delayed dispersal to further predict characteristics of ecological systems that favour weak dispersal-induced synchronization.

Metapopulation theory and the study of fragmentation have emphasized discrete boundaries and movement between populations (Levins 1969, Hanski 1999). The appeal of metapopulation theory lies in its simplicity associated with the assumption of passive and instantaneous population movement between patches. As a result, model predictions applied to natural systems usually ignore the complex landscape matrix that provides the context for discrete populations (Brady et al. 2011, Turner et al. 2001): mountain peaks embedded in valleys, forest patches within meadows and seagrass between coral reefs. Dispersing propagules and migrating individuals can spend significant time and show non random movement within such a landscape matrix depending on the nature of the movement and on distance between populations. Current metapopulation theory assumes that the spatial structure of metapopulations can be captured by a per capita dispersal rate alone, rather than dispersal time.

The study of spatial synchrony is important because of the implications of synchronous fluctuations of abundance on global population persistence: populations oscillating in full synchrony are more susceptible to extinction at low population density because of reduced 'rescue effect' (Brown and Kodric-Brown 1977) from neighbouring patches. Population asynchrony has been associated with increased global population persistence in experiments (Huffaker 1958; Holyoak 2000). 
To better understand the mechanisms leading to synchronization in natural populations, we study the role of weak and delayed dispersal in driving the ecological conditions for the existence and stability of synchronous states, and for their local convergence rate. We illustrate how time delayed dispersal can be used to implement the particulars of individual movement (through active or passive dispersal) in the space between patches. While discrete patch models are useful for studying synchronization of spatially distributed systems from a dynamical systems point of view, they are associated with the assumption of instantaneous dispersal where individuals disperse with no transmission delay. By including a time delay that retains more detailed information about the dispersal process, we extend the patch dynamics model to integrate properties of the landscape matrix that contains discrete populations.

We first formulate an ecological model as a two-patch Rosenzweig-MacArthur predator prey model coupled through prey dispersal with a time delay. We reduce our model to a phase model using weaklycoupled network theory (Appendix A). Our results show that synchronization through weak dispersal is highly sensitive to dispersal time. We find that with slight variation in the delay, the overall influence of weak dispersal on the rate of convergence to synchrony can vary greatly. This means that dispersal time between spatially discrete systems is a key variable for assessing the role of weak dispersal as a cause of synchronous fluctuations in natural systems. We further extend recent results from Goldwyn and Hastings (2008) on synchronization between patches to predict the importance of such a matrix on the synchronization of weakly coupled predator-prey metacommunities.

\section{Ecological model}

Following Goldwyn and Hastings (2008), we use the Rosenzweig-MacArthur predator-prey model for single-patch dynamics:

$$
\begin{aligned}
\frac{d H}{d t} & =r H\left(1-\frac{H}{K}\right)-\frac{a c P H}{b+H} \\
\frac{d P}{d t} & =\frac{a P H}{b+H}-m P .
\end{aligned}
$$

Here $H(t)$ and $P(t)$ represent the size of the prey and predator populations respectively. Prey growth is modeled by logistic growth with intrinsic rate $r$ and carrying capacity $K$, and the intake of prey by predators is modelled with a Holling Type II functional response specified by the parameters $a$ and $b$ and where the conversion ratio of loss of prey to increase in predators is $c>1$. The predators have a linear mortality rate, with parameter $m$.

Now suppose we have two identical and spatially discrete predator-prey patches, each with dynamics following (2.1). We consider the case where only prey disperse between patches with a fixed dispersing rate $D$. The dispersing prey take some finite transmission time $s$ to cross the space between the patches, and this parameter can be derived using from details of individual movement during dispersal (see Appendix D for details). Our delayed-dispersal predictions are compatible with previous theories of time delayed dispersal in two patch models (Prasad et al. 2008), and are shown to more broadly integrate existing spatial ecological frameworks for spatial dynamics (see Appendix D).

If we further assume the absence of growth or mortality during dispersal, we can equate the incoming flux of individuals to a patch at time $t$ with the outgoing flux of individuals at the opposite patch at a time $t-s$, before the present time. This assumption allows us to incorporate the dispersal process into our four-equation model (predator and prey populations in 2 patches) with a simplified prey dispersal term $D\left(H_{j}(t-s)-H_{i}(t)\right)$ in patch $i$. This assumption of no birth or mortality during dispersal is not necessary for our analysis in this section or in Section 3 however, it is crucial for our numerical computation of the function $H$ (Section 4).

The result is the following two-patch Rosenzweig-MacArthur predator-prey model coupled through prey dispersal with a time delay:

$$
\begin{aligned}
\frac{d H_{i}}{d t} & =r H_{i}\left(1-\frac{H_{i}}{K}\right)-\frac{a c P_{i} H_{i}}{b+H_{i}}+D\left(H_{j}(t-s)-H_{i}(t)\right), \\
\frac{d P_{i}}{d t} & =\frac{a P_{i} H_{i}}{b+H_{i}}-m P_{i}, \quad i, j=1,2 ; i \neq j .
\end{aligned}
$$


This is identical to the model studied by Goldwyn and Hastings (2008), except for the time delay of $s$ time units that we include in the dispersal term, and that we allow only one of the species to disperse between patches.

We nondimensionalize 2.2 following Goldwyn and Hastings (2008) to reduce the number of parameters in a way that will be useful later for adjusting the timescale separation of the predator and prey growth rates. Thus we work with the following resulting equivalent form:

$$
\begin{aligned}
\frac{d h_{i}}{d t} & =\frac{1}{\varepsilon}\left(h_{i}\left(1-\alpha h_{i}\right)-\frac{p_{i} h_{i}}{1+h_{i}}\right)+d\left(h_{j}(t-\tau)-h_{i}(t)\right), \\
\frac{d p_{i}}{d t} & =\frac{p_{i} h_{i}}{1+h_{i}}-\mu p_{i}, \quad i, j=1,2 ; i \neq j
\end{aligned}
$$

where we reuse the variable $t$ for scaled time at, and make the following substitutions: $h_{i}=H_{i} / b, p_{i}=[a c / r b] P_{i}, \tau=a s, \alpha=b / K, \mu=m / a, \varepsilon=a / r, d=D / a$.

The single-patch dynamics of the Rosenzweig- MacArthur model are well-understood (Goldwyn and Hastings 2008). There is a region of $\varepsilon-\alpha-\mu$ parameter space in which the dynamics produce a stable limit cycle, namely: $\alpha<1$ and $\mu<\frac{1-\alpha}{1+\alpha}$. This is the case we are interested in for studying synchronization dynamics.

Furthermore, we are concerned with the part of $\varepsilon-\alpha-\mu$ parameter space where the predator-prey oscillations are relaxation-like, since this leads to faster convergence to synchrony when the patches are weakly coupled, a requirement for weak dispersal to be the cause of synchronization in nature. Goldwyn and Hastings (2008) explains how the separation of timescales between the predator and prey leads the single-patch system to relaxation-like oscillations: in the nondimensionalized form $(2.3)$, this is achieved by decreasing any one of $\varepsilon, \alpha$, or $\mu$. Decreasing $\varepsilon$ increases the rate of intrinsic prey growth relative to that of the predator; decreasing $\alpha$ increases the carrying capacity for prey, enhancing the size and time between prey outbreaks; decreasing $\mu$ means slower predator mortality, which leads to longer times for the predator population to decrease to the point where the prey population spikes, so that these spikes happen less frequently (Goldwyn and Hastings 2008). In effect, the prey populations spend more time at low numbers and then grow more rapidly at spikes when $\varepsilon, \alpha$, and $\mu$ are small.

\section{Phase model}

While non-delayed phase model reduction has been used in previous studies of predator-prey dynamics (Goldwyn and Hastings 2008, Goldwyn and Hastings 2011), our implementation of weakly connected network theory to reduce a time-delayed predator-prey system to a phase model is new. The only assumptions required are that coupling between stable limit-cycle oscillators is weak (i.e., $\delta$ is small; we follow Goldwyn and Hastings 2008 and assume $\delta=0.001$ corresponds to weak coupling), and that the explicit time delay $\tau$ is on the order of magnitude of the period of the oscillation, or less. In other words, the delay must not be larger than roughly 10 times a period of oscillation. The latter assumption is likely to apply to natural systems, where dispersal time is limited by individual lifespan.

Suppose we choose parameters $\varepsilon, \alpha$, and $\mu$ for our model (2.3) so that the identical uncoupled subsystems (i.e., the two patches with $\delta=0$ ) oscillate on an exponentially orbitally stable limit cycle $\gamma(t)$, which means that solutions starting close enough to the limit cycle approach it exponentially fast in the limit as time goes to infinity. The coupling in 2.3 is symmetric, so the system can be written in the general form for weakly connected networks as follows:

$$
\begin{aligned}
& \dot{X}_{1}(t)=F\left(X_{1}(t)\right)+\delta W\left(X_{1}(t), X_{2}(t-\tau)\right) \\
& \dot{X}_{2}(t)=F\left(X_{2}(t)\right)+\delta W\left(X_{2}(t), X_{1}(t-\tau)\right),
\end{aligned}
$$

where

$$
\begin{aligned}
& X_{i}=\left(h_{i}, p_{i}\right)^{T}, \\
& F\left(X_{i}\right)=\left(\frac{1}{\varepsilon}\left(h_{i}\left(1-\alpha h_{i}\right)-\frac{p_{i} h_{i}}{1+h_{i}}\right), \frac{p_{i} h_{i}}{1+h_{i}}-\mu p_{i}\right)^{T}, \\
& W\left(X_{i}(t), X_{j}(t-\tau)\right)=\left(h_{j}(t-\tau)-h_{i}(t), 0\right)^{T} .
\end{aligned}
$$


Characterized by its shape, position and natural frequency $\Omega$, each periodic solution $\gamma$ is an isolated closed curve through two-dimensional phase space, a distorted circle that can be parameterized with a phase variable $\theta$ that increases by $2 \pi$ in one period (see Appendix A).

When we consider the full system, the fact that the $\gamma$ are exponentially orbitally stable limit cycle attractors means that for small $\delta$, coupling between the $X_{i}$ only significantly affects the phase variables $\theta_{i}$. Our other required assumption is that the time delay $\tau$ is of an order of magnitude of $2 \pi / \Omega$ or less. Then, by the theorem in Appendix A we can reduce (3.1) to the corresponding two-dimensional phase model:

$$
\begin{aligned}
& \frac{d \theta_{1}}{d t}=\Omega+\delta H\left(\theta_{2}-\theta_{1}-\Phi\right) \\
& \frac{d \theta_{2}}{d t}=\Omega+\delta H\left(\theta_{1}-\theta_{2}-\Phi\right),
\end{aligned}
$$

where $\Phi=\Omega \tau \bmod 2 \pi$ and

$$
H(x)=\frac{1}{T} \int_{0}^{T} \hat{\gamma}(t) \cdot W(\gamma(t), \gamma(t+x / \Omega)) d t
$$

with $\hat{\gamma}(t)$ solving the system

$$
\begin{gathered}
\frac{d \hat{\gamma}(t)}{d t}=-D F(\gamma(t))^{T} \hat{\gamma}(t) \\
\hat{\gamma}(t) \cdot \gamma^{\prime}(t)=1 .
\end{gathered}
$$

Importantly, we see that the explicit time delays present in (3.1) appear in the phase model as simple phase shifts in the coupling functions, which are more mathematically tractable than differential delay equations. Briefly (see Hoppensteadt and Izhikevich 1997 for full proof), this works because time-delayed terms in the phase model approximation to 3.1 show up on the order of $\delta^{2}$, which we consider negligible in weakly connected systems.

Finally, we define the phase difference variable $\phi(t)$ as $\phi \equiv \theta_{1}-\theta_{2}$. This reduces the system 3.2 further, to

$$
\frac{d \phi}{d t}=G(\phi):=\delta[H(-\phi-\Omega \tau)-H(\phi-\Omega \tau)] \equiv \delta H_{\text {delay }}(\phi)
$$

where we just denote $\Phi$ with $\Omega \tau$.

\section{Phase model analysis}

So far we have reduced the full system (3.1) to a one-dimensional dynamical system with variable $\phi$ that is directly related to synchrony: (3.5) relates the parameters $\varepsilon, \alpha$, and $\mu$ using $\Omega$ and $H$, as well as a time delay $\tau$, to the relative phase positions of the two oscillators in their limit cycle. Now suppose the dynamics $\phi(t)$ from any starting phase difference $\phi\left(t_{0}\right)$ converge with time to some fixed phase difference $\phi^{*}$ with $G\left(\phi^{*}\right)=0$. We refer to this phenomenon as phase locking, and say that the system is in-phase synchronized when phase-locked at $\phi^{*}=0$, and asynchronized when phase locked at $\phi^{*} \neq 0$. We also use the term anti-phase synchronization for systems phase locked at $\phi^{*}=\pi$ specifically.

We solve for $H$ numerically using the numerical software XPPAUT (Ermentrout 2002). We first find the numerical solution of a single patch model with given parameters $\varepsilon, \alpha$, and $\mu$. We then calculate $\Omega$, and the function $\hat{\gamma}(t)$ that solves (3.4) (the iPRC, discussed in Appendix A). XPPAUT can then approximate the corresponding coupling function $H(x)$ (the calculation uses $(3.3)$ ). We obtain this function via its first eleven Fourier coefficients $a_{n}$ and $b_{n}, n=0, \ldots, 10$, which approximate

$$
H(x)=\sum_{n=0}^{10}\left[a_{n} \cos (n x)+b_{n} \sin (n x)\right] .
$$

Now by a simple calculation (as done in Kobelevskiy (2008)), we define

$$
\begin{aligned}
H_{\text {delay }}(\phi) & =H(-\phi-\Omega \tau)-H(\phi-\Omega \tau) \\
& =-2 \sum_{n=0}^{10} \sin (n \phi)\left[a_{n} \sin (n \Omega \tau)+b_{n} \cos (n \Omega \tau)\right],
\end{aligned}
$$




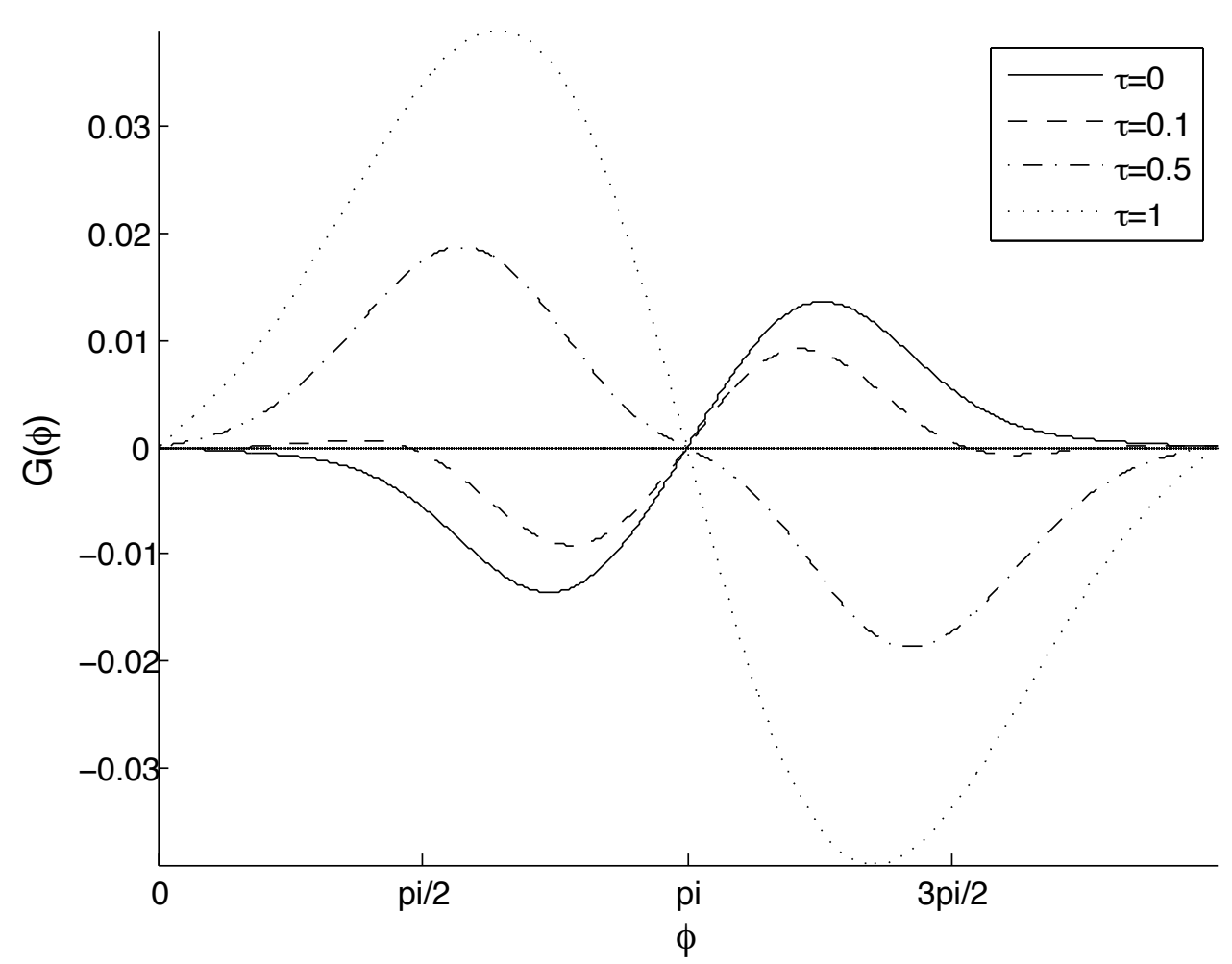

Fig. $1 \varepsilon=0.1, \alpha=0.35, \mu=0.3$, and $\delta=0.001 . G(\phi)$ for a few different values of $\tau$.

so that calling $c_{n}=a_{n} \sin (n \Omega \tau)+b_{n} \cos (n \Omega \tau)$, we have an expression for $G(\phi)=\frac{d \phi}{d t}$ :

$$
G(\phi)=-2 \delta \sum_{n=0}^{10} c_{n} \sin (n \phi) .
$$

Our approximation was not improved by using more than eleven Fourier coefficients. We can now integrate $G(\phi)$ in time to find the solution of the system for an arbitrary starting phase difference to investigate the effect of $\tau$ on phase dynamics, or simply plot $G(\phi)$ to determine the phase-locked states and their stability.

\section{Results}

In this section we investigate the effect of the time delay $\tau$ on the phase-locked states of the phase model (3.5) for one particular set of $\varepsilon-\alpha-\mu$ parameters using the numerical techniques of Section 4 . A validation of these results against the dynamics of the full model $(3.1)$ is presented in Appendix B, while the effect of time delay for a range of $\varepsilon-\alpha-\mu$ parameters is considered in Appendix $\mathrm{C}$.

\subsection{Phase-locked states}

We begin by investigating the effects of a time delay for the parameter set $\varepsilon=0.1, \alpha=0.35$, and $\mu=0.3$ in $(2.3)$, which for an uncoupled patch (i.e., $\delta=0$ ) leads to stable oscillatory dynamics with frequency $\Omega=0.856$. We use $\delta=0.001$ for weak coupling. We retrieve the coefficients $a_{n}$ and $b_{n}$ to construct $G(\phi)=\frac{d \phi}{d t}$ from 4.1 , which has roots corresponding to steady states. Notice that $G(\phi)$ is $T$-periodic in $\tau$. Thus we examine a range of $\tau$ values from 0 to $T=7.34=2 \pi / \Omega$. This range is justified under the assumption that $\tau$ is of the same order of magnitude as $T$ or less. If $G^{\prime}\left(\phi^{*}\right)<0$ at a steady-state $\phi^{*}$, then it is stable, while if $G^{\prime}\left(\phi^{*}\right)>0$ then the steady state is unstable.

The number and stability of phase-locked solutions to the two-patch predator-prey system is strongly affected by delayed dispersal (Figure 2). We start with $\tau=0$, equivalent to instantaneous travel between 


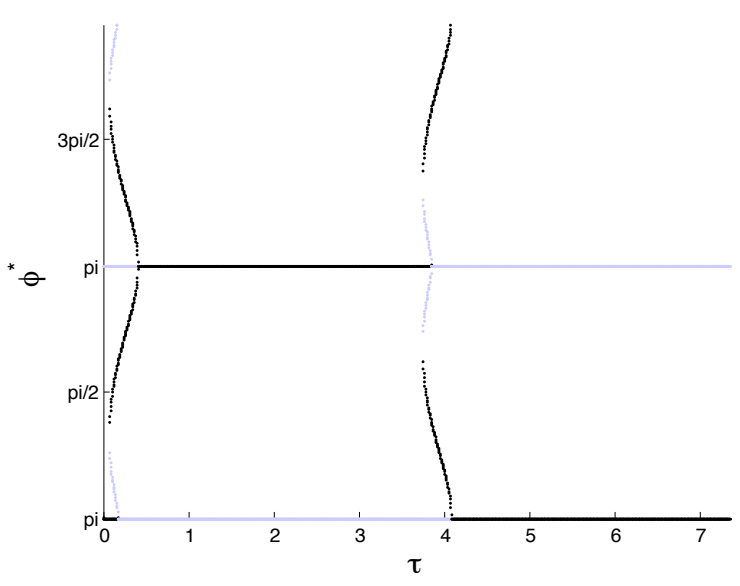

(a)

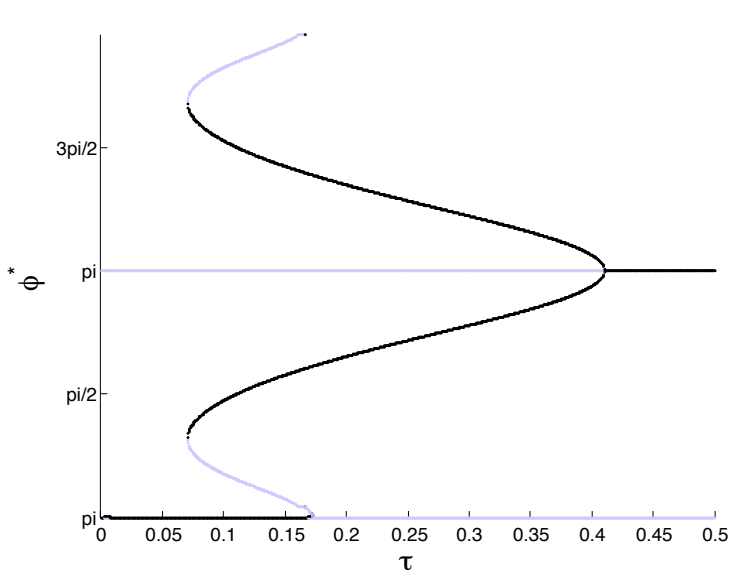

(b)

Fig. $2 \varepsilon=0.1, \alpha=0.35, \mu=0.3$, and $\delta=0.001$. $\tau$-Bifurcation diagram: (a) showing a full $\tau$ range (i.e., over a full period of oscillation), (b) showing values for $\tau$ close to 0 . Stable steady-states are black; unstable steady-states are blue (appearing as grey in monochrome).

patches, and see that the system has one stable steady state solution, $\phi^{*}=0$, and one unstable steadystate solution, at $\phi^{*}=\pi$ (Figures 1 and 2). As $\tau$ is increased, 0 and $\pi$ are always steady-state solutions because $G(\phi)$ is $2 \pi$ periodic and odd. We see that other steady-state solutions may exist, appearing or disappearing in a total of eight saddle-node or pitchfork bifurcations as $\tau$ is varied from 0 to $T=7.34$ (Figure 2).

Our bifurcation analysis reveals the complex response of phase dynamics to a time delay in dispersal. Super-critical pitchfork bifurcations occur at the steady state $\phi^{*}=\pi$ when $\tau=0.41$, and at the steady state $\phi^{*}=0$ when $\tau=4.08$. In the bifurcation at $\tau=4.08$, as can be seen in Figure 2(a), the steady state at $\phi^{*}=0$ changes stability, and a branch of stable steady states bifurcates from $\phi^{*}=0$ with the new stable branch existing for $\tau<4.08$ where the $\phi^{*}=0$ solution is unstable. There is thus a stable steady state at $\phi^{*}=0$ on one side of the bifurcation, and a stable steady state close to 0 on the other side of the bifurcation. Thus at this bifurcation the $\phi^{*}=0$ stable steady-state is carried continuously away from 0 (or the reverse). The behaviour at the other supercritical pitchfork bifurcation at $\phi^{*}=\pi$ when $\tau=0.41$ is similar, except we see that there are actually two branches that bifurcate from $\phi^{*}=\pi$ in symmetric fashion. This is characteristic of all supercritical pitchfork bifurcations (and also occurs in the bifurcation at $\phi^{*}=0, \tau=4.08$ on noting that $\phi=0$ and $\phi=2 \pi$ represent the same phase).

Sub-critical pitchfork bifurcations occur at $\phi^{*}=0$ when $\tau=0.17$ and at $\phi^{*}=\pi$ when $\tau=3.84$ (see Figure 2). These are the most dynamically disruptive type of pitchfork bifurcation, since a stable steady state exists only on one side of these bifurcation points. A system in nature passing through such a bifurcation would 'jump' abruptly from one steady-state to another (outside the region of the bifurcation point).

Saddle-node bifurcations correspond to two steady states (one stable and one unstable) that collide and annihilate so that a stable steady state either appears or disappears. There are four such bifurcations in our system: two at $\tau=0.07\left(\phi^{*}=1.00\right.$ and 5.28) close to the subcritical pitchfork bifurcation at $\tau=0.17$, and two at $\tau=3.74\left(\phi^{*}=2.14\right.$ and 4.15$)$, close to the subcritical pitchfork bifurcation at $\tau=3.84$.

The fact that there are eight bifurcations across the range of $\tau$ where the stable steady states change indicates that the qualitative dynamics of the weakly-connected system, and therefore its ability to synchronize, is remarkably sensitive to the value of the time delay $\tau$. Increasing $\tau$ from 0 to $T$ can produce a full range (i.e., from 0 to $2 \pi$ ) of stable phase differences $\phi^{*}$ (Table 1 ). This enriches our understanding and ability to predict the dynamics of the system beyond the $\tau=0$ case with only one stable steady state (0). When $\tau>0$ it is still possible to have 0 as a stable steady state, but other asynchronous stable steady states are available as well over some values of $\tau$, including anti-phase synchronization at phase difference $\pi$. However, there are also ranges of $\tau$ values where the steady state 0 becomes unstable, and the system is unable to synchronize in-phase (i.e., for the entire interval between $\tau=0.17$ and $\tau=4.08$ ). Overall, our bifurcation diagram (Figure 2 can be used to show that the fraction of $\tau$ values (of the full range length 
Table $1 \varepsilon=0.1, \alpha=0.35, \mu=0.3$, and $\delta=0.001$. Stable steady states for varying $\tau$ values (i.e., on the interval from 0 to a full period of oscillation).

\begin{tabular}{ll}
\hline$\tau$ Range & Stable steady-states \\
\hline$(0,0.07)$ & 0 \\
$(0.07,0.17)$ & $0,0<\phi^{*}<\pi,-\phi^{*}$ \\
$(0.17,0.41)$ & $0<\phi^{*}<\pi,-\phi^{*}$ \\
$(0.41,3.74)$ & $\pi$ \\
$(3.74,3.84)$ & $0<\phi^{*}<\pi, \pi,-\phi^{*}$ \\
$(3.84,4.08)$ & $0<\phi^{*}<\pi,-\phi^{*}$ \\
$(4.08,7.34)$ & 0 \\
\hline
\end{tabular}

$T$ ) where in-phase synchronization is possible (i.e., 0 is a stable steady-state) is $(T+0.17-4.08) / T=0.47$. This means that over half of all possible time delays (on the same order of magnitude as $T$ or less) prevent in-phase synchronization given the parameter values we used in numerical simulations.

In fact, the system loses its ability to synchronize in-phase (i.e., the steady state 0 becomes unstable) even with a very small time delay. If $\tau$ were increased from 0 to just 0.17 (a time delay of $0.17 / \alpha$ in unscaled time), a population with in-phase synchronized dynamics would bifurcate abruptly (through a sub-critical pitchfork bifurcation) to a significantly asynchronous non-zero stable steady state, decreasing the risk of simultaneous extinction in both patches. The phase difference would remain held away from in-phase synchronization as $\tau$ is increased, all the way until $\tau=4.08$. This sensitivity of the dynamics to small changes of $\tau$ also means that if two patches are synchronized in-phase, their global (simultaneous) risk of extinction can be reduced by a change in the delay $\tau$ of less than $(T+0.17-4.08) / 2=1.72$ to bring the dynamics to a sub-critical pitchfork bifurcation where the system shifts to another non-zero steady state at $T+0.17$ (recalling that the bifurcation diagrams are T-periodic), or to a super-critical pitchfork bifurcation where the steady-state phase difference smoothly increases from zero at $\tau=4.08$.

\subsection{Rate of convergence to synchrony}

Previous studies by Goldwyn and Hastings (2008) have shown that in general, relaxation oscillators converge to steady-state dynamics faster than other types of oscillators (due to their iPRCs having greater maximum magnitudes). We find that within this category of relaxation oscillators, a time delay plays a significant role in further determining how strong a synchronizing force weak dispersal can be. We find that convergence strength to synchrony predicted by the model varies widely over the range of $\tau$ values we consider, and in a way that indicates that the system is highly sensitive to small changes of $\tau$.

The local convergence rate to synchrony of a stable steady-state $\phi^{*}$ is calculated as $\left|G^{\prime}\left(\phi^{*}\right)\right|$, the absolute value of the first derivative with respect to $\phi$ of the function $G(\phi)$, evaluated at $\phi^{*}$; or in other words, the absolute value of the slope of the graph of $G(\phi)$ at the zero $\phi^{*}$ (the slope will be negative if $\phi^{*}$ is a stable steady-state). The greater the local convergence rate to synchrony, the shorter the time for transient dynamics around the steady-state; and the more likely it is to be observed in natural systems exposed to recurring perturbations away from their phase-locked state.

Our analysis shows that weak, non-delayed dispersal is not in itself a strong mechanism of in-phase synchrony in terms of convergence strength, compared to delayed dispersal. This is revealed by the slow convergence to in-phase synchrony at $\tau=0$ compared to those systems with delayed dispersal $(\tau>0)$ that have an in-phase stable equilibrium (e.g. $\tau=5.5$; Figure 3 . More specifically, the rate of convergence to a stable $\phi^{*}=0$, or to the steady state with the smallest phase difference, is significantly faster with than without a time delay (Figure 3). This is because at $\tau=0$ the system is close to the pitchfork bifurcation observed at $\tau=0.17$ where 0 loses its stability, and it is precisely at that bifurcation points that $G^{\prime}(\phi)=0$ and that the convergence rate is minimum. Local convergence rate is fastest at the centres of the intervals between consecutive bifurcations points: these are the $\tau$ values where weak dispersal is able to best produce synchronized dynamics in nature. Because there is a bifurcation very close to $\tau=0$, a slight increase in $\tau$ from zero not only forces the system into its asynchronous and eventually anti-phase synchronous phase-locking possibilities, but also increases the rate of convergence to these phase-locked states, and thus the predicted ability of weak dispersal to explain synchrony in nature. Thus, we have shown that for a specific set of parameters corresponding to oscillatory dynamics of a single predator- 


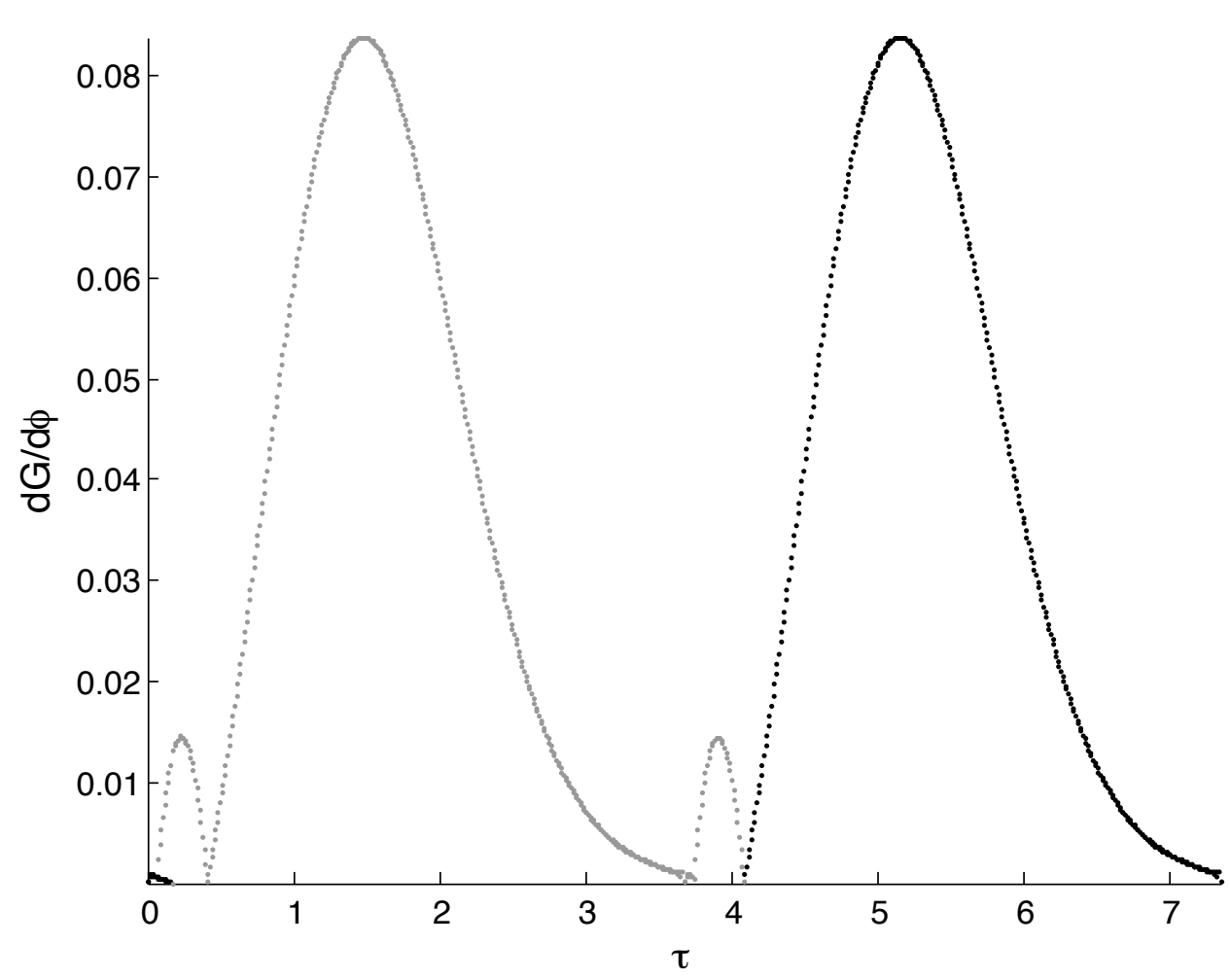

(a)

Fig. $3 \varepsilon=0.1, \alpha=0.35, \mu=0.3$, and $\delta=0.001$. Rate of convergence to stable steady-state 0 (black) or the next stable steady state $\phi^{*}>0$ (gray) if 0 is unstable. The values are shown for the full $\tau$ range (i.e., over a full period of oscillation).

prey patch, even a very small time delay can produce dynamics that are markedly different from the predictions of the non-delayed model.

\section{Discussion}

The importance of a landscape matrix in controlling movement of individuals and propagules between discrete populations has led to its integration into recent management and conservation practices, but most theoretical frameworks of patch dynamics are still based on instantaneous dispersal. Our time-delay approach to modeling dispersal in a patch model constitutes a simple way to incorporate details of individual movement through the landscape matrix within a patch dynamics framework. We show that our patch model with delayed dispersal provides a useful approximation of dispersal through continuous landscapes. Our results show that dispersal time is a spatial process deserving of attention when considering synchronization dynamics. We more precisely show how delayed dispersal can greatly limit the ability of weak dispersal to synchronize predator-prey dynamics to in-phase dynamics, which put a system at highest risk of global extinction. Instead, delayed dispersal is predicted to promote asynchronous phaselocked dynamics across discrete populations. Our results have important implications for understanding causes of spatial synchrony in natural systems and for bridging gaps between simple patch models and landscape ecology.

6.1 Knowing when to be discrete, and when to be continuous

Patch dynamics with delayed dispersal can provide a very useful tool to approximate complex movement patterns across natural landscape matrices. This approach effectively embeds a patch model onto a more concrete physical space resembling a fragmented landscape, where local patches are separated by significant space that individuals disperse across. The modeling technique for dispersal here is applicable 
to any such system in nature where patches are not directly next to each other (in which case $\tau$ would just be zero), even if there's only a small transmission time between patches: there is no limit in general to how sensitive a system can be to small transmission delays.

The notion of time delays in dispersal can be extended to systems involving egg banks, seed banks, dia-pausing eggs, or dormant stage in general. It is usually thought that a dormant stage is a means of "dispersal through time" to increase survival through harsh conditions (Callaghan and Karlson 2002). However, many examples of dormant stages also involve spatial aggregation and thus movement between dormant and non-dormant stages (Rothhaupt 2000). Such situations where dormant propagules disperse through space are just an extreme example of the complex ways that dispersal through space and time are coupled and can explain important ecological phenomena such as persistence and coexistence through storage effects (Chesson 2000). Our work shows indeed that spatial segragation comes hand in hand with temporal segregation, which provides a relevant and insightful approach to modeling population dynamics in spatial systems.

\subsection{Time-delayed dispersal and synchrony}

Identifying causes of synchrony remains a major challenge in many biological systems. Ecologists have provided both theoretical and empirical evidence for weak dispersal as an important cause of spatial synchrony between discrete populations (see Liebhold 2004 for a review), but the question remains: when can weak dispersal be the mechanism behind in-phase synchronization between natural populations that are typically exposed to frequent phase perturbations and embedded within complex landscapes? Goldwyn and Hastings (2008) showed that a requirement for weak dispersal to cause synchronization in a network of predator-prey patches in nature is that the predator-prey dynamics behave as a relaxation oscillator. Relaxation oscillations result from a separation of temporal scales between predator and prey dynamics and lead to fast convergence time to in-phase steady state synchrony. Within this region of parameter space, our study provides a new perspective on the ability of weak dispersal to cause synchronization across natural landscapes where dispersal between habitats is delayed.

Our time-delayed dispersal approach gives a way of using information we may have about a system connected through weak dispersal (the distance between patches, speed of the dispersers, etc.) to infer the importance of weak dispersal as an important factor behind synchronized dynamics. Our results show that the delay itself can have a number of implications for phase dynamics: systems may be carried away from in-phase synchronization and held at a non-zero phase-locking state. Over a range of delay values $(\tau)$ the system may increase from one to three the number of stable phase-locked steady states, further reducing the parameter space resulting in in-phase synchronization. The two patch RosensweigMacArthur model studied here has been associated with bifurcation to anti-phase synchrony in relation to delayed coupling (Prasad et al 2008). Understanding how anti-phase synchrony arises as a mode of dynamics in natural ecological systems is of increasing interest, given its observation in natural systems as a striking phenomenon with significant ecological implications (see Grenfell et al. 2001, He and Stone 2004, Beninca et al. 2009, Koelle and Vandermeer 2004, for examples). Our analysis predicts the general response of phase synchrony (including anti-phase synchrony) to changes in dispersal delay between patches. Our results will contribute to the applicability of phase-dynamical theories (Goldwyn and Hastings 2008) in natural and experimental ecosystems.

Our results also relate the value of $\tau$ to the rate of convergence to in-phase synchrony and show how a time delay can greatly impede the ability of weak dispersal to explain the maintenance of in-phase synchrony in nature, and instead enhance asynchronous and anti-phase synchronous dynamics. The rate of convergence is important because in nature where systems frequently experience perturbations due to stochastic fluctuations in the environment, their steady state dynamics are unlikely to be observed unless they recover quickly relative to the frequency of perturbations. In agreement with more general bifurcation theories (Strogatz 2000), our results show that the local convergence rate is minimum near $\tau$ bifurcation thresholds, and is maximum between these bifurcation points. This is especially relevant because for a broad range of $\varepsilon-\alpha-\mu$ values (see Appendix C), in-phase synchrony bifurcates to phase locking at delay values very close to $\tau=0$. Instantaneous dispersal dynamics is thus highly sensitive to the introduction of arbitrarily small dispersal time.

Our results suggest that the combined knowledge of all stable phase-locked states and of their convergence strength is important to predict when weak dispersal can be a very important cause of syn- 
chronization for systems characterized by relaxation oscillations. An example of such a system are those involving insect outbreaks, which commonly produce large amplitude oscillations (Peltonen et al. 2002). In communities characterized by small amplitude and sinusoidal oscillations, such as in the hare-lynx system, weak dispersal is an unlikely cause of synchronization, and extra information about the transmission delay may not be useful. Such systems are more likely to be synchronized through the Moran effect (Goldwyn and Hastings 2008), or by strong dispersal as dispersal strength decreases convergence time. (The local convergence rate $\left|G^{\prime}\left(\phi^{*}\right)\right|$ in our model inherits the factor $\delta$ from $G(\phi)$ - see (3.5) or 4.1) and so the local convergence rate is proportional to $\delta$ and the convergence time is proportional to $1 / \delta$.)

\subsection{Patch dynamics within landscape matrices}

Fragmented landscapes are typically formed of habitats, connecting corridors, and the overall landscape matrix (Turner et al. 2001). Dispersal time captures a number of landscape properties: spatial arrangement of habitats, the presence and effectiveness of corridors, and the resistance of the landscape matrix to movement (Koh et al 2010). The idea of movement time (time-delay) between habitat fragments as assumed in our study can inform on the role of habitat corridors in connecting wildlife refuges. The use of these corridors in fragmented habitats to assist the dispersal of endangered species and decrease the risk of regional extinction is still controversial (Brady et al. 2011), and could be resolved through the understanding of dispersal delays across landscapes.

While the presence of a landscape matrix and of corridors involve a finite dispersal time between habitats, it can also affect demographic processes and behavior of dispersing individuals, as well as regulate the rate of dispersal and modulate the coupling strength between habitats (Koh et al 2010). The phase model we adopted allows studying movement time within a patch dynamical framework, but it also assumes weak dispersal and no change in individual density or behavior during dispersal. Future work should investigate the importance of a transmission delay with stronger dispersal coupling using delay-differential equations (our model validation in Appendix B outlines one such analytical method). The integration of demographic processes such as mortality during dispersal would also improve the relevance of patch dynamical models for understanding synchrony across complex landscapes. We hope our modeling approach can integrate these more realistic assumptions that are key to conservation, while still contributing to a more general theory of patch dynamics.

Acknowledgements E. Wall is grateful to the McGill University Biology Department for a Science Undergraduate Research Award (SURA). F. Guichard and A.R. Humphries thank the Natural Sciences and Engineering Research Council of Canada (NSERC) for funding though the Discovery Grants Program.

\section{References}

1. Allen LJS (1983) Persistence and Extinction in Lotka-Volterra Reaction-Diffusion Equations. Math Biosci, 65(1):1-12. doi: 10.1016/0025-5564(83)90068-8

2. Beninca E, Johnk KD, Heerkloss R, Huisman J (2009) Coupled predatorprey oscillations in a chaotic food web. Ecol Lett 12:13671378. doi: 10.1111/j.1461-0248.2009.01391.x

3. Blasius B, Tönjes R (2007) Predator-prey oscillations, synchronization and pattern formation in ecological systems. In: L. Schimansky-Geier, B. Fiedler, J. Kurths, E. Schöll (eds) Analysis and Control of Complex Nonlinear Processes in Physics, Chemistry and Biology. World Scientific, Singapore, pp 397-427

4. Brady MJ, McAlpine CA, Possingham HP, Miller CJ, and Baxter GS (2010) Matrix is important for mammals in landscapes with small amounts of native forest habitat. Landscape Ecology, 26(5):617-628. doi: 10.1007/s10980-011-96026

5. Bresloff PC and Lai YM (2013, in press) Dispersal and noise: Various modes of synchrony in ecological oscillators. J Math Bio, doi: 10.1007/s00285-012-0607-9

6. Brown JH, Kodric-Brown A (1977) Turnover rates in insular biogeography: effect of immigration on extinction. Ecol, $58: 445449$

7. Byers JA (2001) Correlated random walk equations of animal dispersal resolved by simulation. Ecol, 82(6):1680-1690. doi: $10.2307 / 2679810$

8. Callaghan T and Karlson R (2002) Summer dormancy as a refuge from mortality in the freshwater bryozoan plumatella emarginata. Oecologia, 132(1):51-59

9. Campbell SA and Kobelevskiy I (2012) Phase models and oscillators with time delayed coupling. Discrete and Continuous Dynamical Systems, 32(8):2653-2673

10. Chesson P (2000) Mechanisms of maintenance of species diversity. Annu Rev Ecol Syst, 31:343

11. Dhamala M, Jirsa V, and Ding M (2004) Enhancement of neural synchrony by time delay. Phys Rev Lett, 92(7):074104 
12. Durrett R, Levin S (1994) The importance of being discrete (and spatial). Theo Popul Biol, 46:363-394. doi: $10.1006 /$ tpbi.1994.1032

13. Ermentrout GB (1994) In: Ventriglia F (ed) Neural Modeling and Neural Networks. Pergamon Press, Oxford

14. Ermentrout GB (2002) Simulating, Analyzing, and Animating Dynamical Systems. SIAM

15. Goldwyn EE, Hastings A (2008) When can dispersal synchronize populations?. Theo Popul Biol, 73:395-402. doi: 10.1016/j.tpb.2007.11.012

16. Goldwyn EE, Hastings A (2011) The roles of the Moran effect and dispersal in synchronizing oscillating populations. Theo Popul Biol, 289:237-246. doi: 10.1016/j.jtbi.2011.08.033

17. Grenfell BT, Bjrnstad ON, Kappey J (2001) Travelling waves and spatial hierarchies in measles epidemics. Nature 414:716723. doi:10.1038/414716a

18. Hanski I (1999) Metapopulation ecology. Oxford University Press, Oxford.

19. He D, Stone L (2003) Spatio-temporal synchronization of recurrent epidemics. Proc R Soc Lond B 270:15191526. doi: $10.1098 / \mathrm{rspb} .2003 .2366$

20. Holyoak M (2000) Habitat patch arrangement and metapopulation persistence of predators and prey. Am Nat, $156: 378389$

21. Hoppensteadt FC, Izhikevich EM (1997) Weakly Connected Neural Networks. Springer-Verlag, New York

22. Huffaker C (1958) Experimental studies on predation: dispersion factors and predatorprey oscillations. Hilgardia, $27: 343383$

23. Izhikivech EM (2008) Phase models with explicit time delays. Phys Rev E, 58:905-908. doi: 10.1103/PhysRevE.58.905

24. Kobelevskiy I (2008) Bifurcation analysis of a system of Morris-Lecar neurons with time delayed gap junctional coupling. Masters Dissertation, University of Waterloo, Canada

25. Koelle K, Vandermeer J (2005) Dispersal-induced desynchronization: from metapopulations to metacommunities. Ecol Lett, 8:167175. doi:10.1111/j.1461-0248.2004.00703.x

26. Koh LP, Lee TM, Sodhi NS, Ghazoul J (2010) An overhaul of the species-area approach for predicting biodiversity loss: incorporating matrix and edge effects. J Appl Ecol, 47(5):1063-1070. doi:10.1111/j.1365-2664.2010.01860.

27. Kuramoto Y (1984) Chemical Oscillations, Waves, and Turbulence. Springer-Verlag, Berlin

28. Levins R (1969) Some demographic and genetic consequences of environmental heterogeneity for biological control. Bulletin of the Entomological Society of America, 15:237-240

29. Liebhold A, Koenig W, Bjørnstad O (2004) Spatial synchrony in population dynamics. Annu Rev Ecol Evol Syst, 35:467-490. doi: 10.1146/annurev.ecolsys.34.011802.132516

30. MATLAB R2012a, The MathWorks Inc., Natick, MA, USA (2012).

31. Moran PAP (1953) The statistical analysis of the Canadian lynx cycle. II. Synchronization and metereology. Aust J Zool, 1:291-298

32. Othmer HG, Dunbar SR, Alt W (1988) Models of dispersal in biological systems. J Math Ecol, 26(3):263-98. doi: 10.1007/BF00277392

33. Peltonen M, Liebhold A, Bjornstad O, and Williams D (2002) Spatial synchrony in forest insect outbreaks: Roles of regional stochasticity and dispersal. Ecology, 83(11):3120-3129

34. Prasad A, Dana SK, Karnatak R, Kurths J, Blasius B, Ramaswamy R (2008) Universal occurrence of the phase-flip bifurcation in time-delay coupled systems. Chaos, 18(2):023111. doi: 10.1063/1.2905146

35. Ranta E, Kaitala V, Lundberg P (1998) Population variability in space and time: the dynamics of synchronous populations. Oikos, 83:376-382. doi: 10.2307/3546852

36. Rothhaupt K (2000) Plankton population dynamics: food web interactions and abiotic constraints. Freshwater Biol, 45(2):105-109

37. Schuster, H and Wagner P (1989) Mutual entrainment of 2 limit-cycle oscillators with time delayed coupling. Prog Theor Phys, 81(5):939-945

38. Strogatz SH (2000) Nonlinear dynamics and chaos with applications to physics, biology, chemistry, and engineering. Westview Press, Cambridge, Mass

39. Turchin P (1998) Quantitative analysis of movement : measuring and modeling population redistribution in animals and plants. Sinauer Associates, Sunderland, Mass

40. Turner MG, Gardner RH, O'Neill RV (2001) Landscape ecology in theory and practice: pattern and process. Springer, New York

41. Winfree AT (1980) The Geometry of Biological Time. Springer, New York

\section{Appendix A}

For ease of analysis in studying synchronization with the assumption of weak dispersal, we reduce the full two-patch model 2.3p to a phase model using concepts from weakly connected network theory (see (Hoppensteadt and Izhikevich 1997)). Here we expand on the phase model reduction used in Section 3. to understand how the general principles of weakly connected network theory can be used on a general system viewed from a coupled oscillator perspective. A weakly connected network in general is any system of the form

$$
\frac{d X_{i}}{d t}=F_{i}\left(X_{i}\right)+\delta W_{i}\left(X_{1}, \ldots, X_{n}\right), \quad i=1, \ldots, n,
$$

where each $X_{i}(t) \in \mathbb{R}^{m}$ and $\delta$ is a small parameter. (For now we do not consider a time delay in coupling.) We are interested in the case where each decoupled subsystem $(\delta=0)$

$$
\frac{d X_{i}}{d t}=F_{i}\left(X_{i}\right), \quad i=1, \ldots, n
$$


has an exponentially orbitally stable limit cycle attractor $\gamma_{i} \subset \mathbb{R}^{m}$.

The limit cycle $\gamma_{i}$ being a periodic orbit of the system, has an associated period $T_{i}$ and a natural frequency $\Omega_{i}=2 \pi / T_{i}$. As a closed curve through $m$-dimensional space, each $\gamma_{i}$ can be parameterized with a phase variable $\theta_{i}$ that increases by $2 \pi$ in one period. With an arbitrary starting point $p_{i} \in \gamma_{i}$, we define a mapping $P_{i}:[0,2 \pi) \rightarrow \mathbb{R}^{m}$ that takes $\theta_{i} \in[0,2 \pi)$ to the unique corresponding point on $\gamma_{i}$ that is $P_{i}\left(\theta_{i}\right)=Y_{i}\left(\theta_{i} / \Omega_{i}\right)=Y_{i}\left(\theta_{i} T_{i} / 2 \pi\right)$, where $Y_{i}(t) \in \mathbb{R}^{m}$ solves $d Y_{i}(t) / d t=F_{i}\left(Y_{i}(t)\right)$ with $Y_{i}(0)=p\left(Y_{i}(t)\right.$ is on the limit cycle $\gamma_{i}$ ) (Hoppenseadt and Izhikevich 1997).

The fact that the $\gamma_{i}$ are exponentially orbitally stable limit cycle attractors means that for small $\delta$, coupling between the $X_{i}$ only significantly affects the phase variables $\theta_{i}$. For systems where oscillators have identical frequencies $\Omega_{1}=\cdots=\Omega_{n}=\Omega$, Malkin's Theorem says that solutions of the system (A.1) can be continuously mapped to solutions of the following canonical phase model defined on the $n$-torus $\mathbb{T}^{n}=\mathbb{S}^{1} \times \cdots \times \mathbb{S}^{1}$ (Hoppensteadt and Izhikevich 1997):

$$
\frac{d \theta_{i}}{d t}=\Omega+\delta H_{i}\left(\theta_{1}-\theta_{i}, \ldots, \theta_{n}-\theta_{i}\right)+\mathcal{O}\left(\delta^{2}\right), \quad i=1, \ldots, n,
$$

where $H_{i}$ are phase coupling functions,

$$
H_{i}\left(\theta_{1}-\theta_{i}, \ldots, \theta_{n}-\theta_{i}\right)=\frac{1}{T} \int_{0}^{T} \hat{\gamma}_{i}(t) \cdot W_{i}\left(\gamma_{1}\left(t+\left(\theta_{1}-\theta_{i}\right) / \Omega\right), \ldots, \gamma_{n}\left(t+\left(\theta_{n}-\theta_{i}\right) / \Omega\right)\right) d t
$$

and $\hat{\gamma}_{i}(t)$ solves the system

$$
\begin{gathered}
\frac{d \hat{\gamma}_{i}(t)}{d t}=-D F\left(\gamma_{i}(t)\right)^{T} \hat{\gamma}_{i}(t) \\
\hat{\gamma}_{i}(t) \cdot \gamma_{i}{ }^{\prime}(t)=1 .
\end{gathered}
$$

The function $\hat{\gamma}_{i}(t)$ is referred to as the infinitesimal phase response curve (iPRC) (Winfree 1980; Kuramoto 1984). It measures the degree to which an arbitrarily short and infinitesimally small perturbation advances (positive valued) or slows (negative valued) the phase (Goldwyn and Hastings 2008). The iPRC can be thought of as a measure of the sensitivity of the oscillator to perturbations at each time $t$ in $\left[0, T_{i}\right)$ : at times when the oscillator is affected most by perturbations (from dispersal), the iPRC has a greater maximum magnitude. Goldwyn and Hastings (2008) show that the greater the separation in predatorprey timescales (i.e., the more relaxation-like the oscillator), the greater the sensitivity of the oscillator to perturbations, and the higher the maximum magnitude of the prey component in the iPRC (Table 2). This explains why relaxation oscillators converge faster to synchrony than more sinusoidal oscillators. It also underlies the requirement for separated timescales between predator and prey for weak coupling to result in synchronization in natural systems (Goldwyn and Hastings 2008).

Crucially, phase model reduction is also possible for weakly connected systems involving an explicit dispersal delay. The phase model for such systems is derived in (Hoppensteadt and Izhikevich 1997) and (Ermentrout 1994). For weak coupling and $\mathcal{O}(1)$ delays (i.e., delays of the same order of magnitude as the period of oscillation or less), the delays do not explicitly appear in the phase model, but rather result in an additional phase shift. The main theorem of weakly connected delayed systems is stated as follows, adapted from (Hoppensteadt and Izhikevich 1997) and (Izhikevich 2008):

Theorem (Phase model with delayed coupling): Consider a weakly connected oscillatory network that has an explicit transmission delay, described by the system

$$
\frac{d X_{i}}{d t}=F_{i}\left(X_{i}\right)+\delta W_{i}\left(X_{1}\left(t-\eta_{i 1}\right), \ldots, X_{n}\left(t-\eta_{i n}\right) ; \quad X_{i} \in \mathbb{R}^{m}, \quad i=1, \ldots, n,\right.
$$

where the $\eta_{i j}$ are finite nonnegative real numbers, all $\mathcal{O}(1)$. Suppose that each uncoupled system has an exponentially orbitally stable T-periodic limit cycle solution $\gamma_{i} \subset \mathbb{R}^{m}$. Then, the system can be reduced to the phase model

$$
\frac{d \theta_{i}}{d t}=\Omega+\delta H_{i}\left(\theta_{1}-\theta_{i}-\Phi_{1 i}, \ldots, \theta_{n}-\theta_{i}-\Phi_{1 i}\right)+\mathcal{O}\left(\delta^{2}\right), \quad i=1, \ldots, n,
$$

where $\Phi_{j i}=\Omega \eta_{j i}$ mod $2 \pi$, and the $H_{i}$ functions are defined by A.4. 


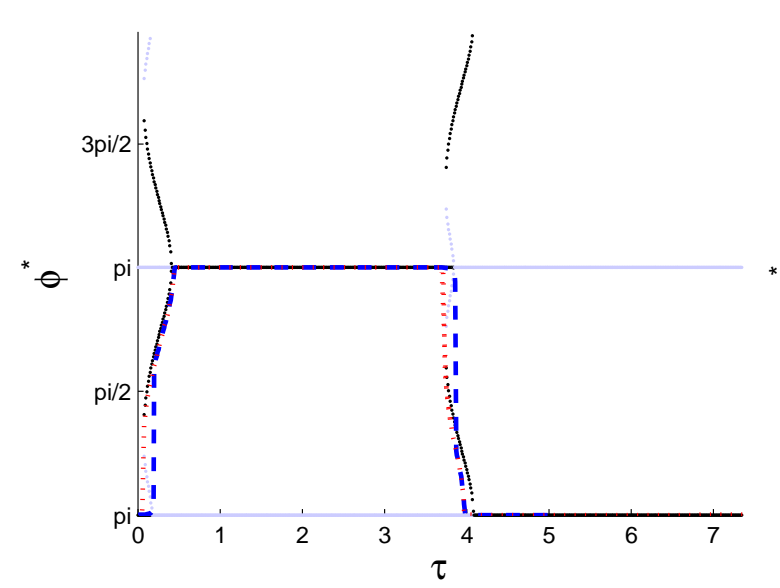

(a)

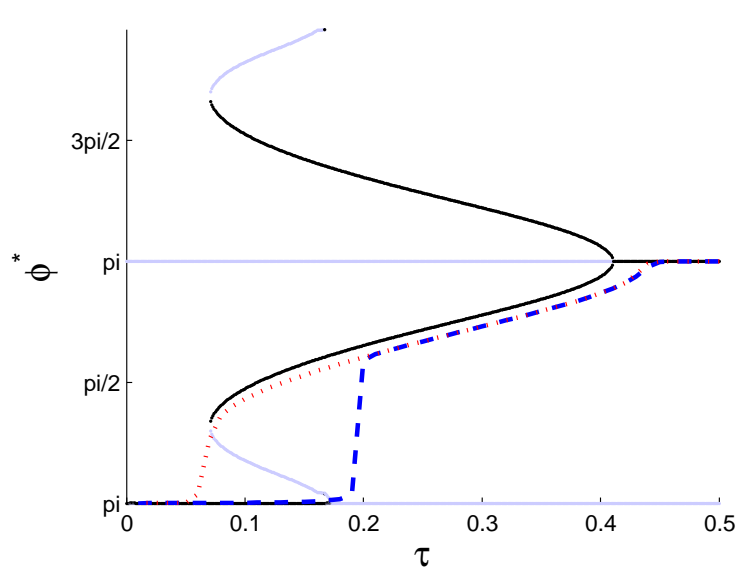

(b)

Fig. 4 The $\tau$-Bifurcation diagram for $\varepsilon=0.1, \alpha=0.35, \mu=0.3$, for the phase reduced model 3.5 as in Figure 2 with superimposed numerically computed phase differences for the full model 3.1 with $\delta=0.001$ for sequences of increasing (blue dashed line) and decreasing (red dotted line) $\tau$ values.

\section{Appendix B}

According to the phase reduction theory of Appendix A the bifurcation diagram for the reduced system (3.5) in Figure 2 should correspond to the phase-locked states of the full system (3.1) in the limit as $\delta \rightarrow 0$. To validate the theory and our numerical implementation of it, we also integrated the full system (3.1) using the matlab 30 initial value problem delay-differential equation solver dde23. The results for the same parameter values as in Section 5 are shown in Figure 4.

We measured the asymptotic phase difference for the full model (3.1) by integrating the delaydifferential equation over a long time interval, then measuring the asymptotic period of the orbit and phase difference using a Poincaré section to detect successive points where $p_{i}(t)=1$ with $\dot{p}_{i}(t)<0$. These long initial value computations were then repeated over a sequence of $\tau$ values, using the orbit found for one $\tau$ value as the starting point for the transient integration for the next $\tau$ value. This approach is similar to observing the dynamics as the delay $\tau$ is slowly adjusted, and different dynamics can be and are observed depending on whether $\tau$ is increased or decreased.

We found that as $\tau$ is varied the period of the full system (3.1) could vary by up to $4 \%$ from the period of single patch model (2.1) with the same parameter values. From Figure 4(a) we see that the asymptotic phase difference is identical for (3.1) and reduced models (3.5) in the intervals where the in-phase or anti-phase solutions are stable. From Figure 4(b) we see that when the reduced model indicates a stable phase-locked solution with phase $\phi^{*} \in(0, \pi)$ then the full model (3.1) has phase locked solutions with slightly perturbed phase. The perturbation in the asymptotic phase is dependent on the parameter $\delta$ with the results in Figure 2 for the reduced model 3.5 representing the limiting behaviour as $\tau \rightarrow 0$. Consequently, for $\delta>0$ the bifurcations at the ends of these intervals are also perturbed slightly. However, it is very hard to quantify exactly how much these bifurcations are perturbed, since at the bifurcation points the convergence rate to the asymptotic phase is zero and it would be necessary to integrate through an infinite time transient to obtain convergence. This is particularly apparent in Figure $4(\mathrm{~b})$ near the bifurcation at $\phi^{*}=0$ and $\tau=0.17$, where the computed solution for the full model initially only lifts away slowly from the unstable solution $\phi^{*}=0$ for $\tau>0.17$.

Figure 4(b) also displays the ecologically relevant phenomenon of hysteresis. When increasing $\tau$ in small increments the solution of the full model stays at the ecologically dangerous phase locked solution $\phi^{*}=0$ for all $\tau<0.17$, until this solution loses stability in the subcritical pitchfork bifurcation at $\tau=0.17$, whereafter for $\tau>0$ the solution rapidly converges to the other branch of stable phase-locked solutions with $\phi^{*}>0$. On the other hand, if instead we decrease $\tau$ after the anti-phase solution loses stability near $\tau=0.41$ the solution remains in a phase locked state with $\phi^{*}>0$ all the way down to $\tau \approx 0.07$ when this solution is destroyed in a saddle node bifurcation, and the phase rapidly converges to $\phi^{*}=0$. Thus for $\tau \in(0.07,0.17)$ different dynamics are observed for the full system depending on whether $\tau$ is being increased or decreased. But this could already have been inferred from Figure 2, and 
Table 2 Parameter sets

\begin{tabular}{lllll}
\hline $\begin{array}{l}\text { Parameter } \\
\text { set }\end{array}$ & $\varepsilon$ & $\alpha$ & $\mu$ & $\begin{array}{l}\text { iPRC (adjoint) } \\
\text { height }\end{array}$ \\
\hline 1 & 0.1 & 0.35 & 0.2 & $1.8 \times 10^{4}$ \\
2 & 0.1 & 0.4 & 0.15 & $4.5 \times 10^{5}$ \\
3 & 0.1 & 0.45 & 0.1 & $1.0 \times 10^{6}$ \\
4 & 0.1 & 0.45 & 0.2 & 180 \\
5 & 0.1 & 0.3 & 0.3 & 1600 \\
6 & 0.1 & 0.35 & 0.3 & 140 \\
7 & 0.1 & 0.4 & 0.25 & 130 \\
8 & 0.1 & 0.45 & 0.3 & 12 \\
9 & 0.1 & 0.25 & 0.3 & $1.0 \times 10^{5}$ \\
10 & 0.1 & 0.3 & 0.35 & 300 \\
11 & 0.1 & 0.35 & 0.4 & 12 \\
12 & 0.1 & 0.4 & 0.35 & 11 \\
13 & 0.1 & 0.2 & 0.45 & $1.1 \times 10^{4}$ \\
14 & 0.1 & 0.25 & 0.4 & 900 \\
15 & 0.1 & 0.3 & 0.45 & 20 \\
\hline
\end{tabular}

is related to the existence of the branch of unstable phase locked orbits seen for $\tau \in(0.07,0.17)$ in the reduced system, and which also exist in the full system but cannot be found by integration, since they are unstable.

We also validated the numerics with other parameter values and found broadly similar results. As we expected the less relaxation-like the periodic orbit was (the lower the value of iPRC in Table 2) the longer became the transient time that we needed to integrate the full system through to see the asymptotic behaviour. On the other hand, for more relaxation-like oscillators the more sensitive the dynamics became to the coupling parameter $\delta$.

\section{Appendix C}

We examine specifically how varying the parameters $\varepsilon, \alpha$ and $\mu$ might affect the general results we showed for a specific set of parameters leading to oscillatory dynamics of a single predator-prey patch. Table 2 shows the parameter sets, as well as the maximum magnitude of the iPRC at these parameter values. As expected, decreasing $\alpha$ and $\mu$ (we keep $\varepsilon=0.1$ ) increases the height of the iPRC. Figures 5 and 6 show the bifurcation diagrams analogous to Figure 2(a) and Figure 2(b) for a number of other parameter sets. Similarly to the parameter set used for our main analysis, varying $\tau$ across the interval $[0, T]$ changes the dynamics and rates of convergence to steady states as the dynamics bifurcate a number of times. We see that there is a range of parameter space where $\phi^{*}=0$ is stable at $\tau=0$, but very close to a bifurcation, so that with even a slight increase in $\tau$, the system can move to asynchrony with a much faster convergence rate (seen best in Figure 6). Investigating this slightly larger region of $\varepsilon-\alpha-\mu$ phase space indicates that our qualitative results for the first set of parameters are generally comparable to other parameter sets, and that decreasing $\alpha$ and $\mu$ (separating the predator-prey timescales and making the oscillator relaxation-like) mainly has an effect that shows up on the dynamics near $\tau=0$, and the $\tau$ value near midway through the period of oscillation, where $\phi^{*}=\pi$ loses its stability.

\section{Appendix D}

This section is meant to examine in further depth the relationship of our patch dynamics with time delayed dispersal to other spatial dynamical frameworks. Durrett and Levin (1994) showed the importance of choosing an approach to modeling spatially distributed systems- mean field approaches, patch models, reaction-diffusion equations, or interacting particle systems- that captures the right level of spatial detail for understanding population dynamics. The time-delay model we consider (see $(2.2)$ ) can be viewed as a combination of two existing treatments of spatial dynamics distinguished in this work. The system neglecting the time delay (i.e., for $s=0$ in (2.2) ) corresponds to the patch model approach: it groups individuals of a species into discrete patches without any spatially-explicit structure informing where patches are with respect to one another. In these models each patch is treated with a mean-field approach 


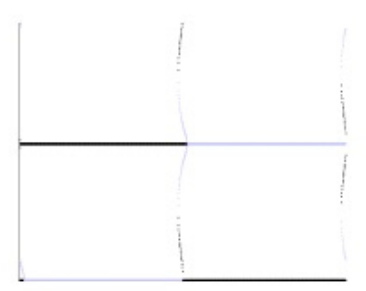

(a) 1

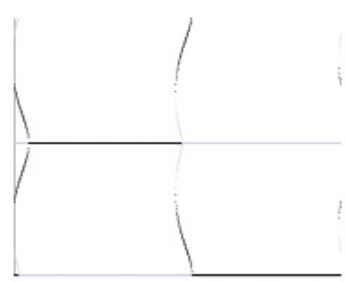

(e) 5

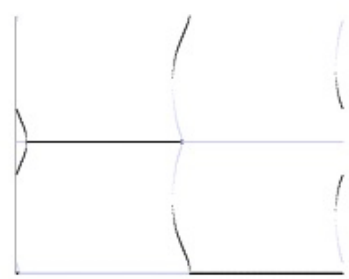

(i) 9

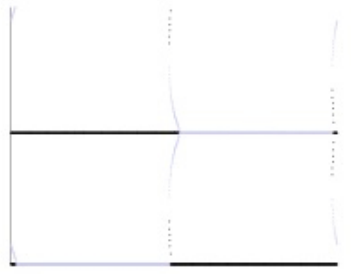

(b) 2

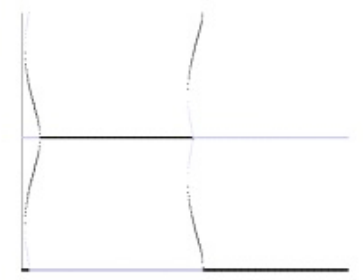

(f) 6

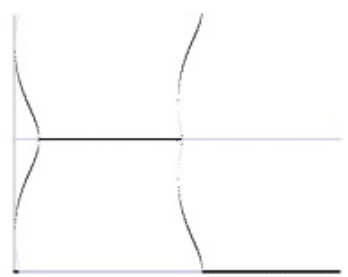

(j) 10

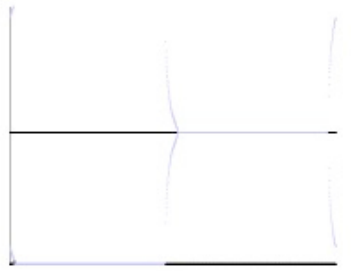

(c) 3

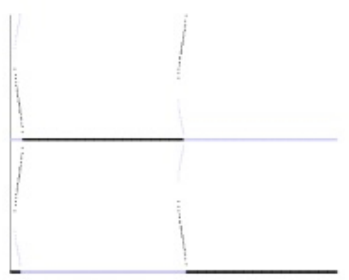

(g) 7

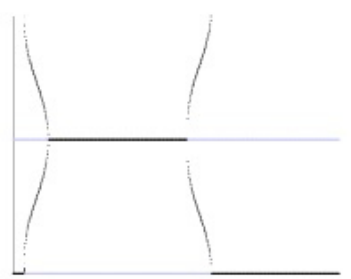

(k) 11

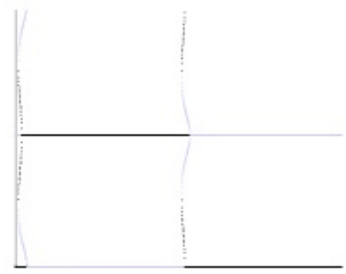

(d) 4

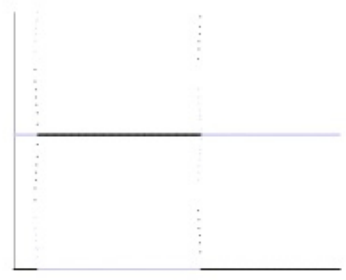

(h) 8

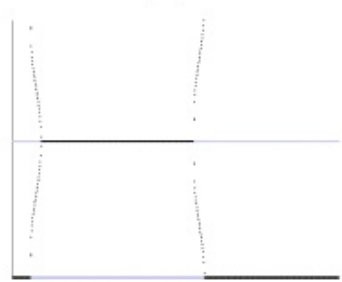

(l) 12

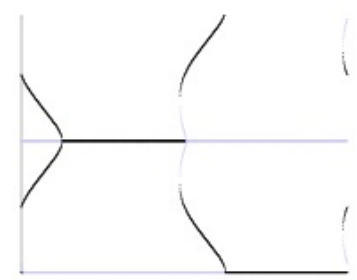

(m) 13

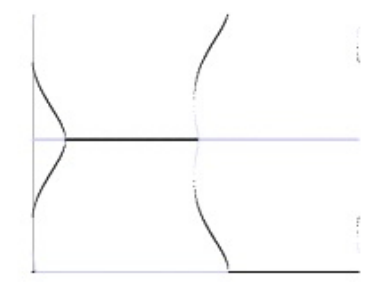

(n) 14

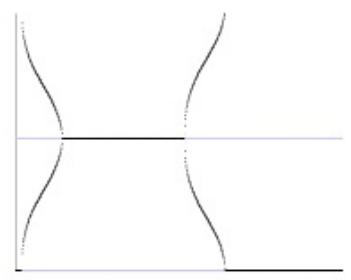

(o) 15

Fig. $5 \delta=0.001$; refer to Table 2 for parameter set values corresponding to numbers 1 through $15 . \tau$ Bifurcation diagrams (full range of $\tau$ from 0 to $T$ ) through a region of $\varepsilon-\alpha-\mu$ parameter space. Stable steady-states are black; unstable steady-states are blue.

where individuals interact equally with each other and the patch itself is described by ordinary differential equations, and patches are connected to all others equally by dispersal or migration at a constant rate (Durrett and Levin 1994).

Patch models and mean-field approaches both lose the level of spatial detail required to account for the movement and local interactions of individuals both in the patch, and in dispersal between patches. The most common approach to modeling spatial systems working on a finer level of spatial detail are reaction-diffusion equations, in which infinitesimal individuals diffuse in space and undergo purely local interactions (reactions). Allen (1983) shows how the dispersal term in a patch model (say, $\left.d\left(h_{j}(t)-h_{i}(t)\right)\right)$ can actually be derived from a simple one-dimensional diffusion equation directly, through the discretization of continuous space and the assumption of Neumann boundary conditions, which confine the individuals to the union of the finite number of patches.

By treating space as continuous, our time-delay model avoids discretizing inter-patch space, keeping closer to the reaction-diffusion equation to describe dispersal in the space between patches. The addition of continuous space between patches (see Figure 7) as opposed to discretizing space allows for handling the concept of diffusive dispersal closer to the more ecologically realistic formulation based on finitespeed random walks in the environment separating the patches, so that dispersers take some meaningful amount of time to cross from patch to patch. The model retains key features of actual movement as it makes a random walk between the patches through the value of the delay $\tau$. Such features are lost in the 


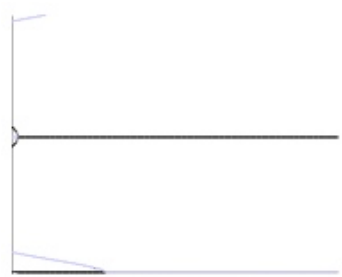

(a) 1

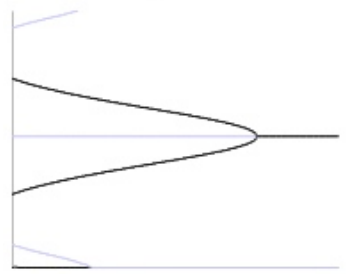

(e) 5

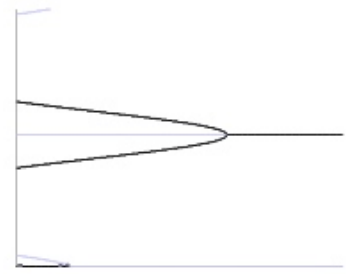

(i) 9

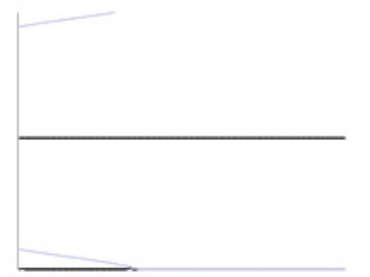

(b) 2

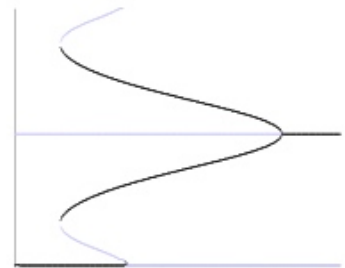

(f) 6

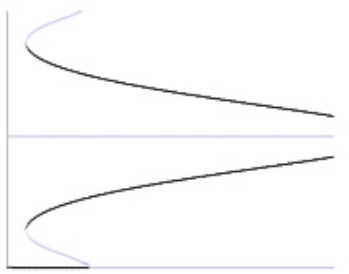

(j) 10

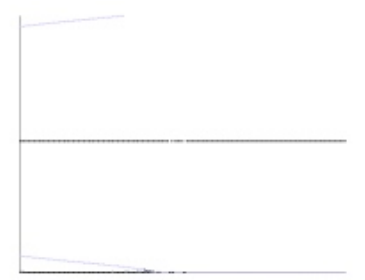

(c) 3

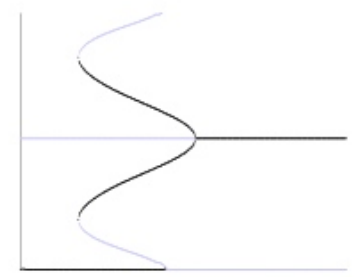

(g) 7

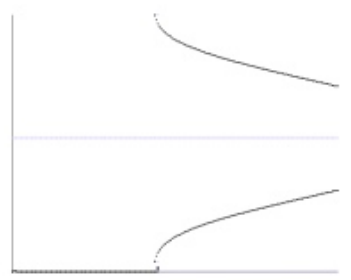

(k) 11

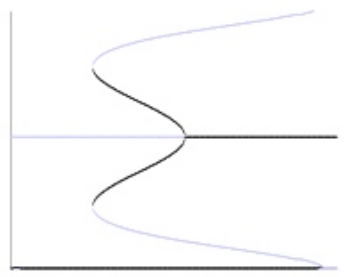

(d) 4

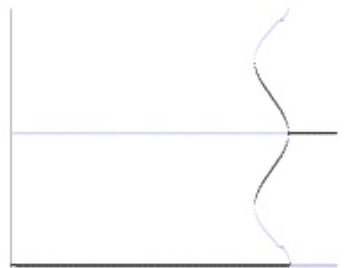

(h) 8

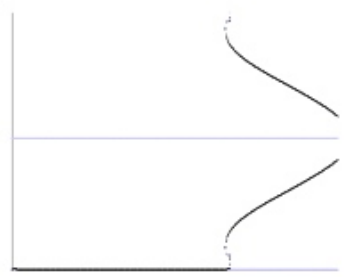

(1) 12

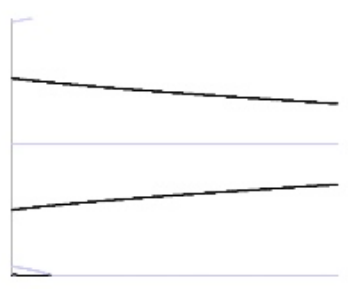

(m) 13

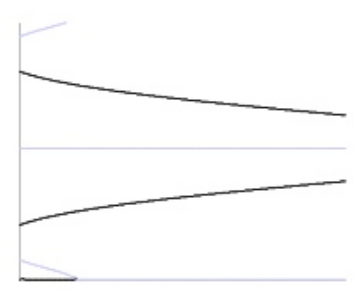

(n) 14

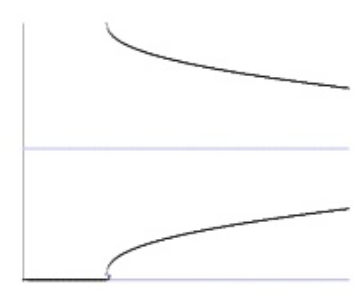

(o) 15

Fig. $6 \delta=0.001$; refer to Table 2 for parameter set values corresponding to numbers 1 through 15. $\tau$ Bifurcation diagrams ( $\tau$ from 0 to 0.5 ) through a region of $\varepsilon-\alpha-\mu$ parameter space. Stable steady-states are black; unstable steady-states are blue.

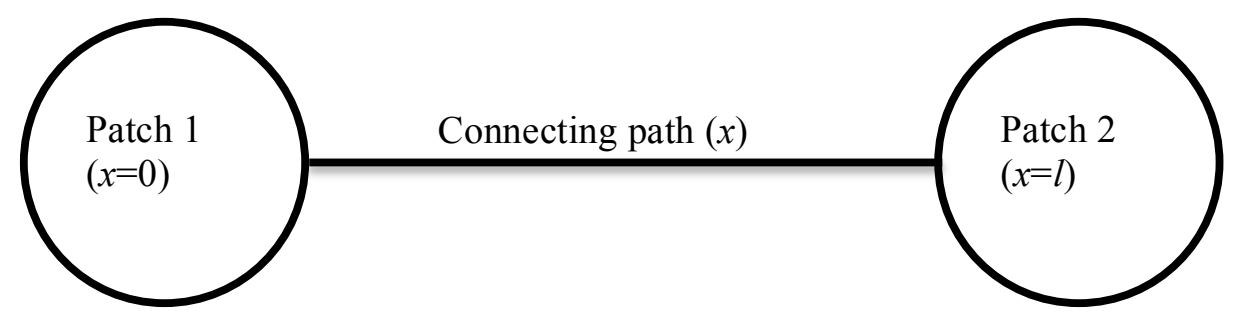

Fig. 7 Two discrete (homogeneous) patches connected by a one-dimensional (two-directional) path for dispersal.

discretized version with no delay. Furthermore, our time-delay dispersal is equivalent to the simplest case possible of the process of individuals reaction-diffusing in space: it assumes a constant mean dispersal duration, and no birth or mortality during dispersal.

In a system of patches connected by dispersal, the mean transmission time $\tau$ can be calculated in several ways using specific details of the individual dispersal process which might be easier to measure in natural systems than the time delay $\tau$ itself. We now demonstrate two such derivations. We start with a continuous space distribution of individuals dispersing in a one-dimensional connecting path between 
patches, according to some underlying assumptions about movement. We then uncover the net squared displacement of individuals in the path, which give the mean time an individual spends dispersing between two patches separated by a distance $l$.

If we first assume individuals move passively at a constant speed $v$ with two-way travel on onedimensional paths connecting patches, we can easily see that the mean crossing time $\tau$ in this case is just $v / l$. Suppose we consider the more general movement pattern integrating active dispersal modeled by the telegraph equation:

$$
\frac{T}{2} \frac{\partial^{2} u}{\partial t^{2}}+\frac{\partial u}{\partial t}=\frac{v^{2} T}{2} \frac{\partial^{2} u}{\partial x^{2}},
$$

where $v$ is the finite speed of individual movement, and $T$ is the characteristic time of movement before a direction reversal (see explanation in Turchin (1998) or derivation in Othmer and Dunbar (1988)). The mean squared displacement over time, $\left|x^{2}(t)\right|$, of a group of individuals instantaneously released into the system at patch 1 is calculated as follows in Othmer and Dunbar (1988):

$$
\left|x^{2}(t)\right|=v^{2} T\left[t-\frac{T}{2}\left(1-e^{-2 t / T}\right)\right] .
$$

While the mean displacement $|x(t)|$ is not quite equal to the square root of the mean squared displacement, one can scale $\sqrt{\left|x^{2}(t)\right|}$ by some factor $C$ to obtain a better estimate for $|x(t)|$ than $\sqrt{\left|x^{2}(t)\right|}$ (Byers 2001). Then the mean displacement is then

$$
|x(t)|=C \sqrt{v^{2} T\left[t-\frac{T}{2}\left(1-e^{-2 t / T}\right)\right]} .
$$

Now set $|x(t)|$ in $(\mathrm{D} .3)$ to $l$ and solve for the corresponding value of time $t$. This defines the delay $s$ which appears in $(2.2)$ as a function of $l, T$, and $v$. Note that the right hand side of (D.3) is a strictly monotonically increasing function of $t$, so there is a unique solution. 